\title{
Analytical Treatment for the Deuteron-Deuteron Interaction
}

\author{
S. S. Avancini, F. F. de Souza Cruz, J. R. Marinelli, D. P. Menezes, and M. M. Watanabe de Moraes \\ Depto de Física - CFM - Universidade Federal de Santa Catarina - Florianópolis - SC - CP. 476 - CEP 88.040 - 900 - Brazil
}

Received on 3 December, 2004

\begin{abstract}
In the study of two interacting systems within the framework of microscopic theories, the correct treatment of the effective interaction between the fragments has been the most troubling problem, due to the technical difficulties in calculating the exchange terms. In the framework of the Generator Coordinate Method, and through a double projection technique, we obtain this effective interaction between two pairs of nucleons, taking into account the Coulomb exchange plus a nuclear part. Our treatment is useful in the analysis of the role of correlated nucleon pairs in photoabsorption experiments by nuclei.
\end{abstract}

Recently, the suggestion that the nuclear response may be interpreted as the response of a collection of neutron-proton pairs (or quasideuterons) was revitalized $[1,2]$ through the introduction of a microscopic method, the so-called Correlated Basis Function method, used to calculate the distribution of quasideuterons in the nuclear system. The adoption of such a point of view makes the problem of finding the effective interaction between the pairs a very important one. In fact, the treatment of systems of interacting particles which are composed of fundamental fermions is an old, but still difficult problem, mainly due to the correct introduction of the Pauli Principle in obtaining the effective potential. As other examples in which this kind of problem could be very appealing, we mention the nucleus-nucleus collision [3] and the formation of compound states from two given fragments.

In this work we present a method, based on the Generator Coordinate Method (GCM), which allows one to obtain the effective ( or collective) potential in such a way that the Pauli Principle is fully taken into account and, at the same time, the microscopic degrees of freedom are kept. Though the GCM was already used in the past for obtaining the phaseshifts in collision problems [4], we show below that it is also possible, using the formalism developed in $[3,5]$, to obtain a complete analytical form for the collective potential for a suitable choice of the basis space. To be specific, we consider the case in which two pairs of nucleons with different isospin quantum numbers interact through a Coulomb plus a nuclear force. Choosing the other appropriate quantum numbers we may particularize to the deuteron-deuteron case.

In what follows we outline the method and the general results. Next, we present our application for the two nucleon pair case and obtain a closed expression for what we call the collective hamiltonian matrix, including the Coulomb and nuclear part. In order to check the consistency of the method and formulae we calculate the $\alpha$ particle binding energy using a simple gaussian potential for the nucleon-nucleon force. Finally, we discuss how to obtain, from the expressions derived here, an analytical form for the potential in the deuterondeuteron case. The final form of the potential and applications will be presented in a separate paper.

The GCM ansatz for a many-body wavefunction of a system of nucleons is defined as: $\left|\psi>=\int f(\vec{\alpha})\right| \vec{\alpha}>d \vec{\alpha}$ and $\left\langle r_{1}, r_{2}, \ldots . r_{N} \mid \psi\right\rangle=\int f(\vec{\alpha})<r_{1}, r_{2}, \ldots|\vec{\alpha}\rangle d \vec{\alpha}$, where $f(\vec{\alpha})$ is a variational function of the generator coordinate $\vec{\alpha}$ and $<r_{1}, r_{2}, \ldots|\vec{\alpha}\rangle$ is a Slater determinant.
By minimizing the energy, we obtain the Griffin Hill Wheeler equation:

$$
\int\left(<\vec{\alpha}|\hat{H}| \vec{\alpha}^{\prime}>-E<\vec{\alpha} \mid \vec{\alpha}^{\prime}>\right) f\left(\vec{\alpha}^{\prime}\right) d \vec{\alpha}^{\prime}=0
$$

As we are interested in the relative motion of two pairs of nucleons, the distance between them $(\vec{\alpha})$, is taken as the generator coordinate. The pair wave function is described by one neutron and one proton in the lowest state of the harmonic oscillator potential. One of the pairs is centered around the point with coordinate vector $\frac{\vec{\alpha}}{2}$ and the other one is centered around $-\frac{\vec{\alpha}}{2}$. The overlap kernel, for our choice for the generator state $|\vec{\alpha}\rangle$, is

$$
\left\langle\vec{\alpha} \mid \vec{\alpha}^{\prime}\right\rangle N\left(\vec{\alpha}, \vec{\alpha}^{\prime}\right)=\left(c_{1} A^{4}+c_{2} B^{4}+c_{12} A^{2} B^{2}\right),
$$

with $A=\exp \left(-\frac{\left(\vec{\alpha}-\vec{\alpha}^{\prime}\right)^{2}}{16 b^{2}}\right), B=\exp \left(-\frac{\left(\vec{\alpha}+\vec{\alpha}^{\prime}\right)^{2}}{16 b^{2}}\right)$. The constants $c_{1}, c_{2}$ and $c_{12}$ depend on the the angular momentum configuration of the system. In the cases we consider here, we always have $c_{1}=c_{2}=1$. The hamiltonian kernel equation (1) can be written as

$$
<\vec{\alpha}|\hat{H}| \vec{\alpha}^{\prime}>=<\vec{\alpha}|T| \vec{\alpha}^{\prime}>+<\vec{\alpha}|V| \vec{\alpha}^{\prime}>
$$

where the kinetic part is:

$$
\begin{gathered}
<\vec{\alpha}|T| \vec{\alpha}^{\prime}>=\left(\frac{\hbar^{2}}{m b^{2}}\right)\left[\frac{3}{4}<\vec{\alpha} \mid \vec{\alpha}^{\prime}>-c_{1} A^{4}\left(\frac{\left(\vec{\alpha}-\vec{\alpha}^{\prime}\right)^{2}}{8 b^{2}}\right)\right. \\
\left.-c_{2} B^{4}\left(\frac{\left(\vec{\alpha}+\vec{\alpha}^{\prime}\right)^{2}}{8 b^{2}}\right)+c_{12} A^{2} B^{2} \frac{\left(\vec{\alpha}^{2}+\vec{\alpha}^{\prime 2}\right)}{8 b^{2}}\right]
\end{gathered}
$$

For the sake of simplicity, from now on, we are going to write the potentials and the kinetic energy kernels in unities of $\frac{\hbar^{2}}{m b^{2}}$. Also, we should stress at this point that the center of mass and internal energy contributions were properly subtracted in the above expression for the kinetic energy. For the potential we have to distinguish two parts. For the Coulomb part we obtain:

$$
<\vec{\alpha}\left|V_{C}\right| \vec{\alpha}^{\prime}>=-\frac{c_{12}}{2} A^{2} B^{2}\left[I\left(\vec{\alpha}+\vec{\alpha}^{\prime}\right)+I\left(\vec{\alpha}-\vec{\alpha}^{\prime}\right)\right]
$$




$$
+A^{4} I\left(\vec{\alpha}+\vec{\alpha}^{\prime}\right)+B^{4} I\left(\vec{\alpha}-\vec{\alpha}^{\prime}\right) .
$$

and for the nuclear part:

$$
\begin{aligned}
<\vec{\alpha}\left|V_{N}\right| \vec{\alpha}^{\prime}> & =2\left(A^{4}+B^{4}\right) I(0)-2 c_{12} A^{2} B^{2}\left(I(\vec{\alpha})+I\left(\vec{\alpha}^{\prime}\right)\right) \\
- & c_{12} A^{2} B^{2}\left[I\left(\vec{\alpha}+\vec{\alpha}^{\prime}\right)+I\left(\vec{\alpha}-\vec{\alpha}^{\prime}\right)\right] \\
& +4 A^{4} I\left(\vec{\alpha}+\vec{\alpha}^{\prime}\right)+4 B^{4} I\left(\vec{\alpha}-\vec{\alpha}^{\prime}\right) .
\end{aligned}
$$

In the above equations we have defined:

$$
I(\vec{\rho})=\frac{1}{b^{3} \sqrt{8 \pi^{3}}} \int \exp \left(-\frac{(\vec{r}-\vec{\rho} / 2)^{2}}{2 b^{2}}\right) V(\vec{r})
$$

with $b$ the oscillator length parameter which defines the potential well. Although the nuclear interaction $V(\vec{r})$ does not contain explicit velocity dependencies, this could be included in the formalism without changing equations 5 and 6 . Also, in the expressions for the potential energy kernel, the terms proportional to $I(0)$ can be recognized as the internal energy potential of the system, i.e., the potential energy between the nucleons pertaining to the same pair. The Griffin Hill Wheeler variational equation can be casted in the Schrodinger form through a double projection that diagonalizes the generator states overlap. The first projection corresponds to a Fourier Transform of the overlap, for which we define the following state

$$
|\vec{K}\rangle=\frac{1}{\sqrt{(2 \pi)^{3} \lambda(K)}} \int e^{i \vec{K} \cdot \vec{\alpha}}|\vec{\alpha}\rangle d \vec{\alpha},
$$

and

$$
\left\langle\vec{K} \mid \vec{K}^{\prime}\right\rangle=\frac{1}{\sqrt{(2 \pi)^{3} \lambda(K)}} \frac{1}{\sqrt{(2 \pi)^{3} \lambda\left(K^{\prime}\right)}} \int d \vec{\alpha} \int d \vec{\alpha}^{\prime} e^{-i \vec{K} \cdot \vec{\alpha}}\left\langle\vec{\alpha} \mid \vec{\alpha}^{\prime}\right\rangle e^{i \vec{K}^{\prime} \cdot \vec{\alpha}^{\prime}}
$$

The $\left\langle\vec{K} \mid \vec{K}^{\prime}\right\rangle$ overlap can then be diagonalized in such a way that: $\int\left\langle\vec{K} \mid \vec{K}^{\prime}\right\rangle \phi_{n}\left(\vec{K}^{\prime}\right) d \vec{K}^{\prime}=\Lambda_{n} \phi_{n}(\vec{K})$. Thus we can define the basis states,

$$
|n\rangle=\int \frac{\phi_{n}\left(\vec{K}^{\prime}\right)}{\sqrt{\Lambda_{n}}}\left|\vec{K}^{\prime}\right\rangle d \vec{K}^{\prime}
$$

such that

$$
\langle n \mid m\rangle=\int d \vec{K}^{\prime} \int d \vec{K} \frac{\phi_{m}^{*}(\vec{K})}{\sqrt{\Lambda_{m}}}\left\langle\vec{K} \mid \vec{K}^{\prime}\right\rangle \frac{\phi_{n}\left(\vec{K}^{\prime}\right)}{\sqrt{\Lambda_{n}}}=\delta_{n m}
$$

Thus we can define the projector as

$$
P=\sum_{n}|n\rangle\left\langle n\left|=\sum_{n} \int d \vec{K}^{\prime} d \vec{K} \frac{\phi_{n}\left(\vec{K}^{\prime}\right)}{\sqrt{\Lambda_{n}}}\right| \vec{K}^{\prime}\right\rangle\langle\vec{K}| \frac{\phi_{n}^{*}(\vec{K})}{\sqrt{\Lambda_{n}}} .
$$

The projected Hamiltonian reads $H_{e f f}=\sum_{n, m}|n\rangle\langle n|\hat{H}| m\rangle\langle m|$. The total projector can be also written as an operator acting on the many body operators through the generator states

$$
P=\sum_{n} \int d \vec{K}^{\prime} d \vec{K} \frac{\phi_{n}\left(\vec{K}^{\prime}\right)}{\sqrt{\Lambda_{n}}}\left|\vec{K}^{\prime}\right\rangle\left\langle\vec{K}\left|\frac{\phi_{n}^{*}(\vec{K})}{\sqrt{\Lambda_{n}}}=\sum_{n} \sqrt{\Lambda_{n}}\right| n\right\rangle \int d \vec{\alpha} \int d \vec{K} \frac{\phi_{n}^{*}(K)}{\sqrt{\lambda(K)}} e^{-i \vec{K} \cdot \vec{\alpha}}\langle\vec{\alpha}|,
$$

and it is easy to prove that the Griffin Hill Wheeler equation is thus equivalent to the Schrödinger type equation for the projected hamiltonian.

$$
\sum_{m}\{\langle n|\hat{H}| m\rangle-E\langle n \mid m\rangle\} \psi_{m}=0
$$

where $\langle n \mid m\rangle=\delta_{n m}$,

Within our choice for the state $|\vec{\alpha}\rangle$, the state $\mid n>$ is:

$$
\left|n>=\frac{1}{(2 \pi)^{3 / 2}} \int d \vec{K} \frac{\phi_{n}(\vec{K})}{\left(\Lambda_{n}\right)^{1 / 2}} \int d \vec{\alpha} e^{-i \vec{K} \cdot \vec{\alpha}}\right| \vec{\alpha}>
$$


$\Lambda_{n}$ is the $\left\langle\vec{K} \mid \vec{K}^{\prime}\right\rangle$ overlap matrix eigenvalue and $\phi_{n}(\vec{K})$ are the corresponding eigenvectors. They are given explicitly by:

$$
\phi_{n}(\vec{K})=\frac{2^{-n / 2}\left(n_{x} ! n_{y} ! n_{z} !\right)^{-1 / 2} b^{3 / 2}}{\pi^{3 / 4}} H_{n_{x}}\left(b K_{x}\right) H_{n_{y}}\left(b K_{y}\right) H_{n_{z}}\left(b K_{z}\right) e^{-(b K)^{2} / 2}
$$

and

$$
\Lambda_{n}=\left(4 \pi b^{2}\right)^{3 / 2} e^{-(b K)^{2}} \lambda_{n}, \quad \lambda_{n}=\left(1+(-)^{n}-c_{12} \delta_{n 0}\right),
$$

where $n=n_{x}+n_{y}+n_{z}$ is the total oscillator quantum number and $H_{n}(x)$ are Hermite polynomials.

After some manipulations we finally obtain for the kinetic and potential matrixes a very compact expression. For the kinetic term:

$$
\begin{aligned}
<n^{\prime}|T| n>=\left(3+\frac{n}{2}\right) \delta_{n n^{\prime}}+ & \sum_{i \neq j \neq k=1}^{3}\left(\frac{\sqrt{\left(n_{i}+2\right)\left(n_{i}+1\right)}}{4} \delta_{n_{i}^{\prime}, n_{i}+2}+\frac{\sqrt{\left(n_{i}^{\prime}+2\right)\left(n_{i}^{\prime}+1\right)}}{4} \delta_{n_{i}, n_{i}^{\prime}+2}\right) \delta_{n_{j}^{\prime}, n_{j}} \delta_{n_{k}, n_{k}} \times \\
& \left(\sqrt{\frac{2\left(1-c_{12}\right)}{2}}\left(\delta_{n 0}+\delta_{n^{\prime} 0}\right)+\delta_{n n^{\prime} \neq 0}\right)-\frac{9}{4} \delta_{n n^{\prime}}
\end{aligned}
$$

where 1,2,3 stand for $x, y, z$ and $\delta_{n n^{\prime}}=\delta_{n_{x} n_{x}^{\prime}} \delta_{n_{y} n_{y}^{\prime}} \delta_{n_{z} n_{z}^{\prime}}$. The symbol $\delta_{n n^{\prime} \neq 0}$ means that it is equal to 1 if $n$ and $n^{\prime}$ are both different from zero and it is 0 otherwise. And for the potential term:

$$
<n^{\prime}|V| n>=\frac{1}{2^{\frac{n+n^{\prime}}{2}}\left(n_{x} ! \ldots n_{z^{\prime}} !\right)^{1 / 2} \lambda_{n}^{1 / 2} \lambda_{n^{\prime}}^{1 / 2}} \frac{1}{(2 \pi)^{3 / 2} b^{3}} \int d^{3} r V(\mathbf{r}) \exp \left(\frac{-r^{2}}{2 b^{2}}\right) I_{C, N}(\mathbf{r})
$$

where for the Coulomb interaction we define:

$$
\begin{gathered}
I_{C}(\mathbf{r})=-\frac{c_{12}}{2} \frac{i^{n+n^{\prime}}}{2^{\left(n+n^{\prime}\right) / 2}}\left((-1)^{n^{\prime}}+1\right) H_{n_{x}+n_{x^{\prime}}} H_{n_{y}+n_{y^{\prime}}} H_{n_{z}+n_{z^{\prime}}}+ \\
+\frac{i^{n+n^{\prime}}}{2^{\left(n+n^{\prime}\right) / 2}}\left((-1)^{n^{\prime}}+1\right) n_{x} ! \ldots n_{z^{\prime}} ! \sum_{k_{1} k_{2} k_{3}}^{\left(n_{x}+n_{x^{\prime}}\right) / 2 \ldots} \frac{2^{2\left(k_{1}+k_{2}+k_{3}\right)}}{k_{1} ! k_{2} ! k_{3} !\left(n_{x}-2 k_{1}\right) ! \ldots\left(n_{z^{\prime}}-2 k_{3}\right) !} H_{n_{x}+n_{x^{\prime}}-2 k_{1}} H_{n_{y}+n_{y^{\prime}}-2 k_{2}} H_{n_{z}+n_{z^{\prime}}-2 k_{3}},
\end{gathered}
$$

and for the nuclear interaction part, we have:

$$
\begin{gathered}
I_{N}(\mathbf{r})=2^{n+1} n_{x} ! n_{y} ! n_{z} !\left((-1)^{n}+1\right) \delta_{n n^{\prime}}-2 c_{12}\left[\frac{i^{n}}{2^{n / 2}} H_{n_{x}} H_{n_{y}} H_{n_{z}} \delta_{n^{\prime} 0}+\frac{i^{n^{\prime}}}{2^{n^{\prime} / 2}} H_{n_{x^{\prime}}} H_{n_{y^{\prime}}} H_{n_{z^{\prime}}} \delta_{n 0}\right]- \\
-c_{12} \frac{i^{n+n^{\prime}}}{2^{\left(n+n^{\prime}\right) / 2}}\left((-1)^{n^{\prime}}+1\right) H_{n_{x}+n_{x^{\prime}}} H_{n_{y}+n_{y^{\prime}}} H_{n_{z}+n_{z^{\prime}}}+ \\
+4 \frac{i^{n+n^{\prime}}}{2^{\left(n+n^{\prime}\right) / 2}}\left((-1)^{n^{\prime}}+1\right) n_{x} ! \ldots n_{z^{\prime}} ! \sum_{k_{1} k_{2} k_{3}} \frac{2^{2\left(k_{1}+k_{2}+k_{3}\right)}}{k_{1} ! k_{2} ! k_{3} !\left(n_{x}-2 k_{1}\right) ! \ldots\left(n_{z^{\prime}}-2 k_{3}\right) !} H_{n_{x}+n_{x^{\prime}}-2 k_{1}} H_{n_{y}+n_{y^{\prime}}-2 k_{2}} H_{n_{z}+n_{z^{\prime}}-2 k_{3}},
\end{gathered}
$$

where the argument of the Hermite polynomials are always $\left\{\frac{x}{\sqrt{2} b}, \frac{y}{\sqrt{2} b}, \frac{z}{\sqrt{2} b}\right\}$, in that order. The formalism shown above can be directly applied to calculate the binding energy of the $\alpha$ particle. This can be done choosing the correct cou- 
pling coefficients. In this case, $c_{12}=1$. Also, we have used a gaussian potential for the nuclear interaction defined as: $V(\mathbf{r})=V_{0} \exp \left(-\frac{r^{2}}{a^{2}}\right)$, with $a=1.5 f m, V_{0}=50 \mathrm{MeV}$ and the oscillator parameter $b$ chosen in such a way to reproduce the root mean square radius. With this parametrization we build and diagonalize the hamiltonian matrix and find the value $-22.5 \mathrm{Mev}$ for the $\alpha$ particle binding energy. Of course, the introduction of the exchange force terms as well as the tensor component in the nuclear potential should be done in order to get a better result. However this simple test can give us a first good check for the model consistency and our formulae. Note that, for the Gaussian and Coulomb potentials, all the remaining integrals which appear in equations (20)and (21) can be done analytically.

Besides the energy and eigenstates it is also possible to obtain an explicit form for the effective potential and inertia, starting from the $H_{e f f}=\sum_{n, m}|n\rangle\langle n|H| m\rangle\langle m|$. For that we can build a collective (effective) momentum representation taking the $\{|K\rangle\}$ vectors, defined above as a basis. Thus the effective Hamiltonian in the momentum representation is given by

$$
\left\langle K\left|H_{e f f}\right| K^{\prime}\right\rangle=\sum_{n, m}\langle K \mid n\rangle\langle n|H| m\rangle\left\langle m \mid K^{\prime}\right\rangle
$$

and, through the fourier transform, we obtain the position representation of the effective hamiltonian

$$
\left\langle X\left|H_{e f f}\right| X^{\prime}\right\rangle=\sum_{n, m} \sqrt{\Lambda_{n} \Lambda_{m}}\langle n|H| m\rangle \tilde{\phi}_{n}(X) \tilde{\phi}_{m}^{*}\left(X^{\prime}\right) .
$$

The collective, or effective hamiltonian can be written as a function of collective momentum and position through a Weyl-Wigner transform or equivalently through an expansion on the non-locality (see details in references [5],[3]):

$$
H_{e f f}=\frac{1}{8}\{P,\{P, B(X)\}\}+V(X)
$$

where the inertia can be written as

$$
B(X)=\frac{1}{M(X)}=\int d \xi \frac{(-i \xi)^{2}}{2 !}\left\langle X+\frac{\xi}{2}|H| X-\frac{\xi}{2}\right\rangle
$$

and the potential as

$$
V(X)=\int d \xi\left\langle X+\frac{\xi}{2}|H| X-\frac{\xi}{2}\right\rangle .
$$

The final form of the potential and inertia are left to a forthcoming paper.

This work was partially supported by CNPq (Brazil).
[1] O. Benhar. A. Fabrocini, S. Fantoni, A. Yu. Illarionov, and G.I. Lykasov, Nucl. Phys. A 703, 70 (2002).

[2] O. Benhar. A. Fabrocini, S. Fantoni, A. Yu. Illarionov, and G.I. Lykasov, Phys. Rev C 67, 014326 (2003).

[3] M.M. Watanabe de Moraes, Tese de Doutorado, IFUSP, 1987; A.F.R. de Toledo Piza and E.J.V. de Passos, Il Nuovo Cim. B 45 1 (1978).
[4] B. Giraud, J.C. Hocquenghem, and A. Lumbroso, Phys. Rev. C 7, 2274 (1973).

[5] E.J.V. Passos and F.F. de Souza Cruz, Phys. Rev. C 24, 690 (1981).

[6] D.A. Zaikin, Nucl. Phys. A 170, 584 (1971). 